\title{
Spin-vortex nucleation in a Bose-Einstein condensate by a spin-dependent rotating trap
}

\author{
Hiroki Chiba and Hiroki Saito \\ Department of Applied Physics and Chemistry, The University of Electro-Communications, Tokyo 182-8585, Japan
}

(Dated: October 27, 2018)

\begin{abstract}
A method to produce a spin-dependent rotating potential using near-resonant circularly polarized laser beams is proposed. It is shown that half-quantum vortices are nucleated in a spinor BoseEinstein condensate with an antiferromagnetic interaction. In contrast to the vortex nucleation in a scalar BEC, the spin-vortex nucleation occurs at a low rotation frequency $\left(\simeq 0.1 \omega_{\perp}\right.$ with $\omega_{\perp}$ being the radial trap frequency) in a short nucleation time $\simeq 50 \mathrm{~ms}$ without dissipation. A method for nondestructive measurement of half-quantum vortices is discussed.

PACS numbers: 03.75.Mn, 03.75.Lm, 37.10.Vz
\end{abstract}

\section{INTRODUCTION}

Quantized vortices are hallmarks of superfluidity and have widely been studied in Bose-Einstein condensates (BECs) of atomic gas. In scalar BECs, vortex states have been generated using a variety of methods, e.g., phase imprinting [1, 2, 3] and potential rotation [4, 5]. Vortices in BECs of atoms with spin degrees of freedom (spinor BECs) have been attracting increasing interest since the Berkeley group [6] recently observed spontaneous creation of polar-core vortices in the magnetization of a spin- ${ }^{87} \mathrm{Rb}$ BEC using a nondestructive spinsensitive imaging technique [7]. In this experiment, spin vortices are created through the dynamical instability arising from ferromagnetic interaction [8]. The MIT group has generated Mermin-Ho and Anderson-Toulouse vortices in a spin-1 ${ }^{23} \mathrm{Na}$ BEC using the Berry phaseimprinting method [9]. These spin-vortex states are predicted to be stable in a rotating potential [10]. However, spin-vortex nucleation by a rotating potential has not yet been realized experimentally.

For a scalar BEC in a rotating potential, there is a critical rotation frequency for the vortex nucleation [4, 11], above which the surface mode becomes dynamically unstable [12, 13], allowing vortices to enter the condensate. According to the numerical simulations in Ref. 14], energy dissipation is needed to reproduce the dynamics of vortex nucleation observed in the experiments.

In the present paper, we propose a method to create spin vortices using a spin-dependent rotating potential, which is produced by near-resonant circularly polarized laser beams. Using this method, we can rotate spin sublevels selectively. We numerically demonstrate that halfquantum spin vortices [15, 16, 17] are nucleated in the antiferromagnetic ground state of the spin- $1{ }^{23} \mathrm{Na}$ BEC. The critical rotation frequency for this spin-vortex nucleation is $\simeq 0.1 \omega_{\perp}$ with $\omega_{\perp}$ being the radial trap frequency, and the nucleation occurs at $t \sim 50 \mathrm{~ms}$ without dissipation. This is in marked contrast to the vortex nucleation in a scalar BEC, in which the critical rotation frequency is $\simeq 0.7 \omega[12,13]$ and vortices are never nucleated for $t \lesssim 100 \mathrm{~ms}$ unless dissipation is taken into account [14].
A method to observe half-quantum vortices in a nondestructive manner is also proposed.

This paper is organized as follows. Section [1] provides a method to create a spin-dependent optical potential. Section [II numerically demonstrates the nucleation of half-quantum vortices in a spin- $1{ }^{23} \mathrm{Na}$ BEC. Section IV discusses a method for nondestructive measurement of half-quantum vortices. Section $\nabla$ presents our conclusions.

\section{SPIN-DEPENDENT OPTICAL POTENTIAL}

It is known that a far-off-resonant laser beam produces a potential that is independent of $m_{F}$ for the hyperfine state $\left|F, m_{F}\right\rangle$ [18]. We show here that an appropriately tuned laser beam can produce a potential that strongly depends on $m_{F}$. For simplicity, we restrict ourselves to alkali atoms with electron spin $S=1 / 2$ and nuclear spin $I=3 / 2$ (e.g., ${ }^{23} \mathrm{Na}$ and ${ }^{87} \mathrm{Rb}$ ).

We consider an atom in the electronic ground state $\left|\mathrm{g}, F=1, m_{F}\right\rangle$ located in circularly polarized laser field with frequency $\omega_{0}$. The energy shift due to the ac Stark effect for the ground state is proportional to

$$
\Delta E \propto \sum_{n} \frac{\left|\left\langle n\left|\hat{\sigma}_{ \pm}\right| \mathrm{g}, F=1, m_{F}\right\rangle\right|^{2}}{\hbar \omega_{0}-E_{n}},
$$

where the summation is taken for the relevant states $|n\rangle$ with energy $E_{n}$. The dipole operators $\hat{\sigma}_{ \pm}$in Eq. (1) change the orbital angular momentum of the outermost electron from $\left|L=0, m_{L}=0\right\rangle$ to $\left|L=1, m_{L}= \pm 1\right\rangle$.

When the laser frequency is close to the $D_{1}$ and $D_{2}$ lines, Eq. (1) is approximated to be

$$
\begin{aligned}
& \Delta E \propto \\
& \frac{1}{\hbar \omega_{0}-E_{\mathrm{D}_{1}}} \sum_{F^{\prime}=1}^{2}\left|\left\langle\mathrm{D}_{1}, F^{\prime}, m_{F} \pm 1\left|\hat{\sigma}_{ \pm}\right| \mathrm{g}, F=1, m_{F}\right\rangle\right|^{2} \\
& +\frac{1}{\hbar \omega_{0}-E_{\mathrm{D}_{2}}} \sum_{F^{\prime}=0}^{3}\left|\left\langle\mathrm{D}_{2}, F^{\prime}, m_{F} \pm 1\left|\hat{\sigma}_{ \pm}\right| \mathrm{g}, F=1, m_{F}\right\rangle\right|^{2},
\end{aligned}
$$


(a) $\mathrm{D}_{1}$ line

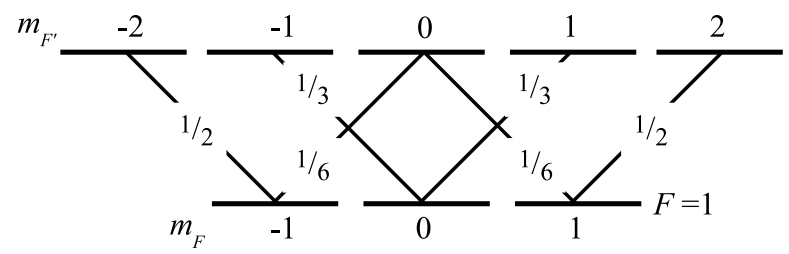

(b) $\mathrm{D}_{2}$ line

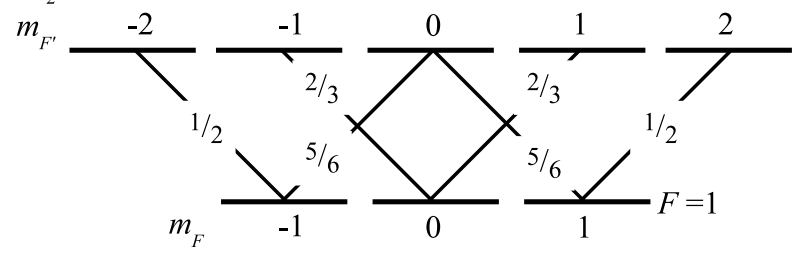

(c) $\hbar \omega_{0}=\left(E_{\mathrm{D} 1}+E_{\mathrm{D} 2}\right) / 2$

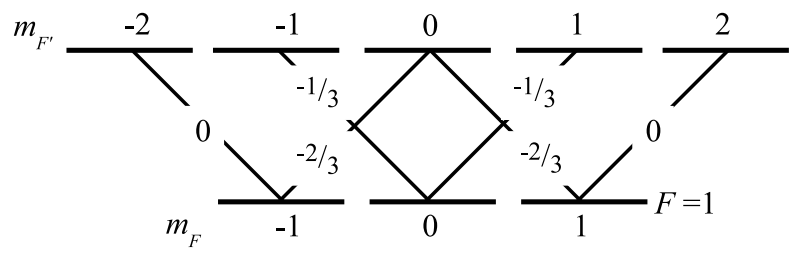

FIG. 1: Transition strengths with circularly polarized laser beams for the (a) $D_{1}$ and (b) $D_{2}$ lines. (c) Subtraction of the transition strengths in (b) from those in (a) for the case of $\hbar \omega_{0}=\left(E_{\mathrm{D}_{1}}+E_{\mathrm{D}_{2}}\right) / 2$.

where $E_{\mathrm{D}_{1}}$ and $E_{\mathrm{D}_{2}}$ are the energies and $\left|\mathrm{D}_{1}, F^{\prime}, m_{F^{\prime}}\right\rangle$ and $\left|\mathrm{D}_{2}, F^{\prime}, m_{F^{\prime}}\right\rangle$ are the excited states for the $\mathrm{D}_{1}$ and $\mathrm{D}_{2}$ lines, respectively. Since $J=1 / 2(J=3 / 2)$ and $I=3 / 2$ for the $\mathrm{D}_{1}\left(\mathrm{D}_{2}\right)$ state, the possible hyperfine spins are $F^{\prime}=1,2\left(F^{\prime}=0,1,2,3\right)$, where $\boldsymbol{J}=\boldsymbol{L}+\boldsymbol{S}$. Calculating each term in Eq. (2), we obtain the transition strengths given in Figs. 1 (a) and 1 (b).

If, in particular, the laser frequency is tuned to the center of the $\mathrm{D}_{1}$ and $\mathrm{D}_{2}$ lines, i.e., $\hbar \omega_{0}=\left(E_{\mathrm{D}_{1}}+E_{\mathrm{D}_{2}}\right) / 2$, the factors before the summations in Eq. (2) have the same magnitude with opposite signs. The transition coefficients in this case are shown in Fig. 1(c). We note that the transition coefficients for $m_{F}= \pm 1 \rightarrow m_{F^{\prime}}= \pm 2$ vanish. This indicates that a $\sigma_{+}\left(\sigma_{-}\right)$laser does not affect the $m_{F}=1(-1)$ state. The produced potential is an attractive potential, since the coefficients are negative. Thus, the $\sigma_{ \pm}$polarized beams with $\hbar \omega_{0}=\left(E_{\mathrm{D}_{1}}+E_{\mathrm{D}_{2}}\right) / 2$ combined with a far-off-resonant beam produce an optical potential $V_{m_{F}}(\boldsymbol{r})$ for each $m_{F}$ state as

$$
\begin{aligned}
V_{0}(\boldsymbol{r}) & =V(\boldsymbol{r})+\frac{1}{2}\left[V_{+}(\boldsymbol{r})+V_{-}(\boldsymbol{r})\right], \\
V_{ \pm 1}(\boldsymbol{r}) & =V(\boldsymbol{r})+V_{\mp}(\boldsymbol{r}),
\end{aligned}
$$

where $V(\boldsymbol{r})$ and $V_{ \pm}(\boldsymbol{r})$ are proportional to the strengths of the far-off-resonant beam and the $\sigma_{ \pm}$polarized beams, respectively.

We estimate the lifetime of the BEC. Since the laser frequency $\omega_{0}=\left(E_{\mathrm{D}_{1}}+E_{\mathrm{D}_{2}}\right) /(2 \hbar)$ is close to the $\mathrm{D}_{1}$ and
$\mathrm{D}_{2}$ transitions, the lifetime of the BEC is shortened by the spontaneous emission. We assume that the dominant contribution to the trapping potential is made by the far-off-resonant beam (with intensity $\propto A_{\text {far }}$ and detuning $\Delta_{\text {far }}$ ), and the $\sigma_{ \pm}$polarized beams (with intensity $\propto A_{\text {near }}$ and detuning $\left.\Delta_{\text {near }}\right)$ are only small perturbations [say, $\left.\left(A_{\text {near }} / \Delta_{\text {near }}\right) /\left(A_{\text {far }} / \Delta_{\text {far }}\right)=0.05\right]$. The ratio of the loss rates then becomes

$$
\frac{A_{\text {near }} / \Delta_{\text {near }}^{2}}{A_{\text {far }} / \Delta_{\text {far }}^{2}}=0.05 \frac{\Delta_{\text {far }}}{\Delta_{\text {near }}} .
$$

In Ref. [19], $\Delta_{\text {far }} \simeq 2 \times 10^{14} \mathrm{~Hz}$, and the loss rate for ${ }^{23} \mathrm{Na}$ atoms is $\simeq 0.03 \mathrm{~Hz}$. Using these parameters, the right-hand side of Eq. (4) is $\simeq 40$, and the lifetime for the present system is estimated to be $\sim 0.8 \mathrm{~s}$.

\section{DYNAMICS OF SPIN VORTEX NUCLEATION}

\section{A. Formulation of the problem}

We employ the zero-temperature mean-field approximation. The dynamics of a BEC for spin-1 atoms are described by the three-component Gross-Pitaevskii (GP) equations given by

$$
\begin{aligned}
i \hbar \frac{\partial \psi_{0}}{\partial t}= & {\left[-\frac{\hbar^{2}}{2 M} \nabla^{2}+V_{0}(\boldsymbol{r}, t)+c_{0} n\right] \psi_{0} } \\
& +\frac{c_{1}}{\sqrt{2}}\left(F_{+} \psi_{1}+F_{-} \psi_{-1}\right), \\
i \hbar \frac{\partial \psi_{ \pm 1}}{\partial t}= & {\left[-\frac{\hbar^{2}}{2 M} \nabla^{2}+V_{ \pm 1}(\boldsymbol{r}, t)+c_{0} n\right] \psi_{ \pm 1} } \\
& +c_{1}\left(\frac{1}{\sqrt{2}} F_{\mp} \psi_{0} \pm F_{z} \psi_{ \pm 1}\right),
\end{aligned}
$$

where $M$ is the mass of an atom, and $\psi_{m}$ describes the mean-field wave functions satisfying

$$
\sum_{m=-1}^{1} \int\left|\psi_{m}\right|^{2} d \boldsymbol{r}=N
$$

with $N$ being the number of atoms. The interaction coefficients $c_{0}$ and $c_{1}$ are given by

$$
c_{0}=\frac{4 \pi \hbar^{2}}{M} \frac{a_{0}+2 a_{2}}{3}, \quad c_{1}=\frac{4 \pi \hbar^{2}}{M} \frac{a_{2}-a_{0}}{3},
$$

where $a_{0}$ and $a_{2}$ are the $s$-wave scattering lengths for colliding channels with total spins 0 and 2 , respectively. The system is ferromagnetic for $c_{1}<0$, and antiferromagnetic or polar for $c_{1}>0$. In Eq. (5), the atomic density $n$ is defined as

$$
n=\sum_{m=-1}^{1}\left|\psi_{m}\right|^{2},
$$


and the magnetization has the forms

$$
\begin{aligned}
& F_{z}=\left|\psi_{1}\right|^{2}-\left|\psi_{-1}\right|^{2}, \\
& F_{+}=F_{-}^{*}=\sqrt{2}\left(\psi_{1}^{*} \psi_{0}+\psi_{0}^{*} \psi_{-1}\right) .
\end{aligned}
$$

In the present analysis, we consider ${ }^{23} \mathrm{Na}$ atoms, which have an antiferromagnetic interaction [20]. We use the scattering lengths $a_{0}+2 a_{2}=53.4 a_{\mathrm{B}}$ [21] and $a_{2}-a_{0}=$ $2.47 a_{\mathrm{B}}$ [22], where $a_{\mathrm{B}}$ is the Bohr radius. For the initial state, we first prepare the antiferromagnetic ground state $\psi_{ \pm 1}=\psi_{\text {ini }}$ and $\psi_{0}=0$ using the imaginary-time propagation method. If $\psi_{0}=0$ in the initial state, the right-hand side of Eq. (5a) vanishes, and $\psi_{0}$ always remains 0 . In realistic situations, however, quantum and thermal fluctuations and residual atoms may trigger the growth of the $m=0$ component. We therefore simulate this possibility by giving the small white noise to the initial state of $\psi_{0}$ as

$$
\psi_{0}(\boldsymbol{r})=\mathcal{N} \epsilon(\boldsymbol{r}), \quad \psi_{ \pm 1}(\boldsymbol{r})=\mathcal{N} \psi_{\mathrm{ini}}(\boldsymbol{r}),
$$

where $\mathcal{N}$ is a normalization constant and $\epsilon(\boldsymbol{r})$ includes the complex random numbers obeying the normal distribution $e^{-|\epsilon|^{2} /\left(2 \sigma^{2}\right)} /\left(2 \pi \sigma^{2}\right)$. The random numbers are set to each point of the numerical mesh. The value $\sigma$ is chosen to be $\sigma=3.5 \times 10^{-3}$, for which the initial population of the $m=0$ component is about $1 \%$.

In the initial-state preparation, only the far-offresonant laser beam is applied, which produces the spinindependent axisymmetric trapping potential,

$$
V(\boldsymbol{r})=\frac{1}{2} M\left[\omega_{\perp}^{2}\left(x^{2}+y^{2}\right)+\omega_{z}^{2} z^{2}\right] .
$$

For $t>0$, we additionally apply a time-dependent $\sigma_{-}$ polarized laser beam, producing a potential rotating at a frequency $\Omega$,

$$
V_{-}(\boldsymbol{r}, t)=-\frac{1}{2} M \omega^{\prime 2} X(t)^{2},
$$

where

$$
X(t)=x \cos \Omega t+y \sin \Omega t .
$$

This potential can be generated using two beams rotating around the center of the trap. In the following calculation, we take $\omega^{\prime 2}=0.05 \omega_{\perp}^{2}$. We do not apply the $\sigma_{+}$ polarized laser beam, i.e.,

$$
V_{+}(\boldsymbol{r})=0 .
$$

The $m=-1$ component therefore does not undergo a rotating potential [see Eq. (3)].

We assume that the trapping potential $V(\boldsymbol{r})$ is tight in the $z$ direction and the system is effectively two dimensional (2D). When $\hbar \omega_{z}$ is much larger than the characteristic energy of the system, the wave function in the $z$ direction is frozen in the ground state of the harmonic potential. The effective 2D interaction strength is obtained by integrating the GP energy functional with respect to $z$.
We use the trap frequencies $\left(\omega_{\perp}, \omega_{z}\right)=2 \pi \times(120,5000)$ Hz.

The time evolution of the system is obtained by numerically solving the 2D GP equation using the finite difference method with the Crank-Nicolson scheme. We divide $38.2 \mu \mathrm{m} \times 38.2 \mu \mathrm{m}$ space into a $200 \times 200$ mesh. We have verified that the results do not depend on the size of the mesh.

\section{B. Nucleation of half-quantum vortices}

Figure 2 shows the time evolution of the density and phase profiles with $N=2 \times 10^{4}$. The rotation frequency of the potential $V_{-}$in Eq. (13) is chosen to be $\Omega=0.13 \omega_{\perp}$. At $t=20 \mathrm{~ms}$, both $m= \pm 1$ components start to deform, and the two topological defects approach the $m=1$ component. At $t=50 \mathrm{~ms}$, the two topological defects enter the $m=1$ cloud, where the density holes in the $m=1$ component are filled with the $m=-1$ component. After that, the topological defects leave the condensate $(t=150 \mathrm{~ms})$. Interestingly, the topological defects enter the condensate again $(t=250 \mathrm{~ms})$, and the entry-exit cycles are repeated. The total density is almost unchanged throughout the dynamics because of $c_{0} \gg c_{1}$. We find that no appreciable spin-exchange dynamics occurs, and the $m=0$ component remains small $(<3 \%)$ for $t<300 \mathrm{~ms}$.

The topological spin structures in Fig. 2 (at $t=50 \mathrm{~ms}$ and $250 \mathrm{~ms}$ ) are the half-quantum vortices [15, 16, 17]. In a spin-1 system, the general form of the half-quantum vortex located at $x=y=0$ is given by [16]

$$
\boldsymbol{\Psi}_{\mathrm{hqv}}=\left(\begin{array}{c}
\psi_{1} \\
\psi_{0} \\
\psi_{-1}
\end{array}\right)=\left(\begin{array}{c}
f_{1}\left(r_{\perp}\right) e^{ \pm i \phi} \\
0 \\
f_{-1}\left(r_{\perp}\right)
\end{array}\right),
$$

where $r_{\perp}=\left(x^{2}+y^{2}\right)^{1 / 2}$ and $\phi=\arg (x+i y)$. The function $f_{1}\left(r_{\perp}\right)$ vanishes at $r_{\perp}=0$ and in a infinite system $f_{1}(\infty)=f_{-1}(\infty)$ should be satisfied. The spatial rotation of $\boldsymbol{\Psi}_{\text {hqv }}$ around the $z$ axis is related to the spin rotation as

$$
e^{-i \hat{L}_{z} \chi} \boldsymbol{\Psi}_{\mathrm{hqv}}=e^{\mp i \chi / 2} e^{\mp i \hat{F}_{z} \chi / 2} \boldsymbol{\Psi}_{\mathrm{hqv}},
$$

where $\hat{L}_{z}=-i \partial_{\phi}, \hat{F}_{z}=m$, and $\chi$ is an arbitrary angle. Equation (17) indicates an interesting fact: when we go around the half-quantum vortex core $(\chi=2 \pi)$, the spin state rotates only by $\pm \pi$. Thus, the half-quantum vortex has a structure similar to a Möbius strip. The spin vortices shown in Fig. 2 have the same topological structure as Eq. (16) in the vicinity of the vortex cores. We can therefore regard the dynamics in Fig. 2 as half-quantum vortex nucleation.

For a scalar BEC, the critical rotation frequency above which vortex nucleation occurs is $\Omega \simeq 0.7 \omega_{\perp}$, and the typical nucleation time is $\sim 100 \mathrm{~ms}$ [11]. In order to reproduce this nucleation time by the GP equation, we must take into account the effect of dissipation [14]. In 


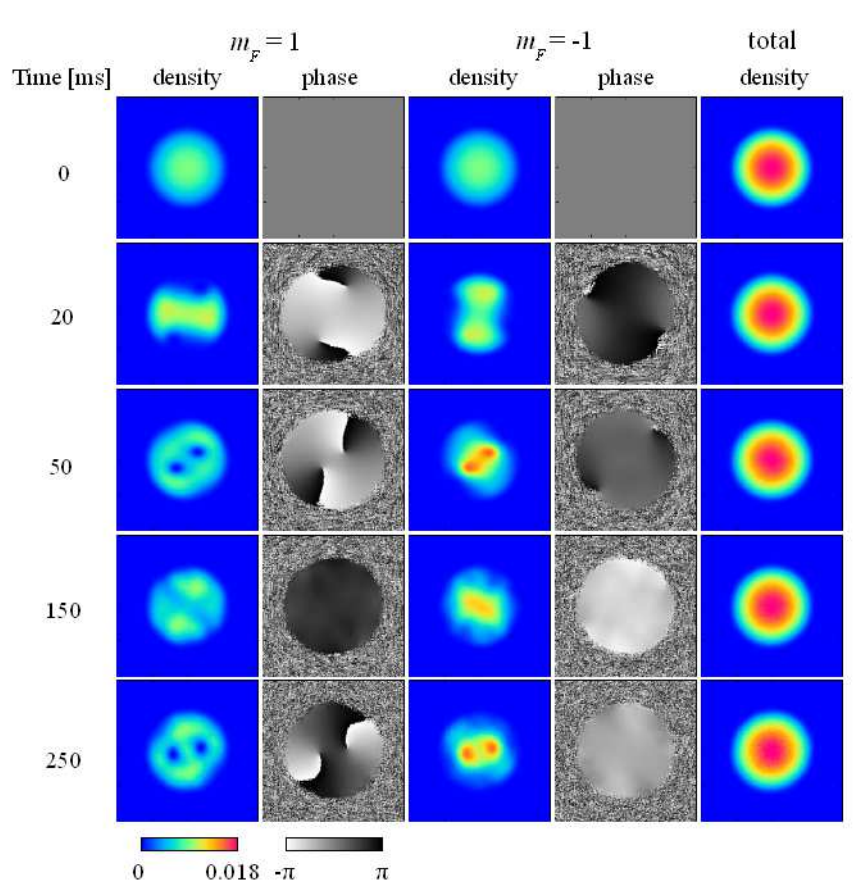

FIG. 2: (Color) Time evolution of the density and phase profiles of the $m= \pm 1$ components and the total density profile for the potential given in Eqs. (12)-(15) with $\Omega=0.13 \omega_{\perp}$. The $m=1$ component is affected by the rotating potential. The $m=0$ component is negligibly small. The field of view of each panel is $38.2 \mu \mathrm{m} \times 38.2 \mu \mathrm{m}$. The unit of the density is $N / a_{\text {ho }}^{2}$ with $a_{\text {ho }}=\left[\hbar /\left(M \omega_{\perp}\right)\right]^{1 / 2}$.

contrast, the spin-vortex nucleation in the present system occurs at much lower rotation frequency $\Omega=0.13 \omega_{\perp}$, and the nucleation time is $t \simeq 50 \mathrm{~ms}$ even without dissipation. These significant differences between the scalar and spinor systems originate from the energy cost for vortex nucleation. For a scalar BEC, the energy cost is determined by $c_{0}$ because of the density hole at the vortex core, whereas the energy cost by the core of the spin vortex is determined by $c_{1}\left(\ll c_{0}\right)$.

Figure 3 shows time evolution of the orbital angular momentum in the $m=1$ component,

$$
L_{1}=-i \int \psi_{1}^{*} \frac{\partial}{\partial \phi} \psi_{1} d \boldsymbol{r}
$$

We note that $L_{1}$ remains small for $\Omega=0.05 \omega_{\perp}$ and $0.5 \omega_{\perp}$, whereas $L_{1}$ becomes large at $\Omega=0.13 \omega_{\perp}$. This implies that there is a region in which the dynamical instability against spin-vortex nucleation sets in. The oscillation of the green curve in Fig. 3 corresponds to the cycles of entry and exit of the half-quantum vortices shown in Fig. 2 .

For $\Omega \gtrsim 0.2 \omega_{\perp}$, we found that the vortices nucleate not only in the $m=1$ component but also in the $m=-1$ component even though $V_{-1}$ is not a rotating potential. This is because the $m=-1$ component effectively feels a rotating potential through the interaction with the ro-

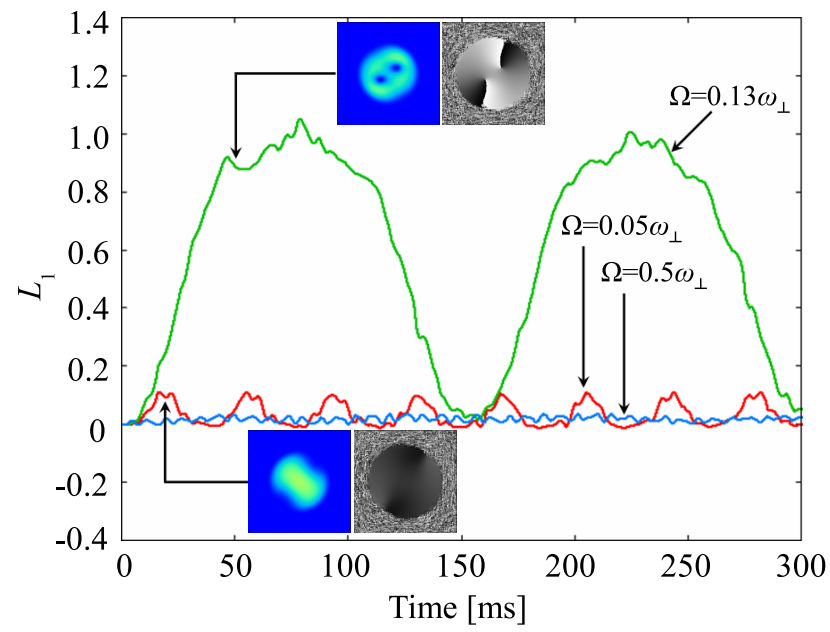

FIG. 3: (Color) Time evolution of the orbital angular momentum $L_{1}$ in the $m=1$ component for $\Omega=0.05 \omega_{\perp}, 0.13 \omega_{\perp}$, and $0.5 \omega_{\perp}$. The insets show the density and phase profiles at 50 $\mathrm{ms}$ for $\Omega=0.13 \omega_{\perp}$ and at $20 \mathrm{~ms}$ for $\Omega=0.05 \omega_{\perp}$.

tating $m=1$ component. By the same mechanism, we can create the vortices only in the $m=-1$ component when $V_{-}$in Eq. (13) is positive. The dependence of the dynamics of the spin-vortex nucleation on the external potential merits further study.

We have considered so far the case of zero magnetic field. When the magnetic field is applied in the $z$ direction, the $m=0$ component is energetically favored and its growth is enhanced. In order to suppress the growth of the $m=0$ component, the magnetic field must be $B \lesssim 10 \mathrm{mG}$. When the magnetic field is $B=10 \mathrm{mG}$ and the initial population of the $m=0$ component is $\simeq 1$ $\%$, the $m=0$ population is suppressed below $3 \%$ for $t<300 \mathrm{~ms}$.

\section{NONDESTRUCTIVE MEASUREMENT OF HALF-QUANTUM VORTICES}

Using the nondestructive spin-sensitive imaging technique developed by the Berkeley group [7], we can observe the half-quantum vortices. In the Berkeley method, a $\sigma_{+}$ circularly polarized probe light is shone in the $y$ direction and phase-contrast images are obtained. The phasecontrast signal is proportional to $A_{0} n+A_{1} F_{y}+A_{2} F_{y}^{(2)}$, where $F_{y}=\left(F_{+}+F_{-}\right) /(2 i)[$ see Eq. (10)] and

$$
F_{y}^{(2)}=\sum_{m, m^{\prime}} \psi_{m}^{*}\left(f_{y}^{2}\right)_{m m^{\prime}} \psi_{m^{\prime}}=\left|\psi_{1}-\psi_{-1}\right|^{2} / 2+\left|\psi_{0}\right|^{2}
$$

with $f_{y}$ being the $y$ component of the spin-1 matrix, and the coefficients $A_{0}, A_{1}$, and $A_{2}$ are given in Tab. [ In the Berkeley experiments [6, 7], the $F=1 \rightarrow F^{\prime}=2 \mathrm{D}_{1}$ transition was used. For a ferromagnetic BEC, the magnitude $\left|F_{+}\right|$and phase $\arg \left(F_{+}\right)$of the transverse magne- 


\begin{tabular}{ccccc}
\hline \hline transition & $A_{0}$ & $A_{1}$ & $A_{2}$ & $R$ \\
\hline$F=1 \rightarrow F^{\prime}=1 \mathrm{D}_{1}$ & $1 / 12$ & $-1 / 24$ & $-1 / 24$ & $2 / 3$ \\
$F=1 \rightarrow F^{\prime}=2 \mathrm{D}_{1}$ & $1 / 4$ & $5 / 24$ & $1 / 24$ & $2 / 13$ \\
$F=1 \rightarrow F^{\prime}=0 \mathrm{D}_{2}$ & 0 & $-1 / 6$ & $1 / 6$ & 2 \\
$F=1 \rightarrow F^{\prime}=1 \mathrm{D}_{2}$ & $5 / 12$ & $-5 / 24$ & $-5 / 24$ & $2 / 3$ \\
$F=1 \rightarrow F^{\prime}=2 \mathrm{D}_{2}$ & $1 / 4$ & $5 / 24$ & $1 / 24$ & $2 / 13$ \\
$F=1 \rightarrow F^{\prime}=3 \mathrm{D}_{2}$ & 0 & 0 & 0 & - \\
\hline \hline
\end{tabular}

TABLE I: Coefficients of the phase-contrast signal $\propto A_{0} n+$ $A_{1} F_{y}+A_{2} F_{y}^{(2)}$ for each transition. Signal-to-bias ratio is proportional to $R=A_{2} /\left(A_{0}+A_{2} / 2\right)$.

tization are obtained from the oscillation of $F_{y}$ due to the Larmor precession.

In the present case, the spin state is written as

$$
\left(\begin{array}{c}
\psi_{1}(\boldsymbol{r}, t) \\
\psi_{0}(\boldsymbol{r}, t) \\
\psi_{-1}(\boldsymbol{r}, t)
\end{array}\right) \simeq\left(\begin{array}{c}
\zeta_{1}(\boldsymbol{r}) e^{-i \omega_{\mathrm{L}} t} \\
0 \\
\zeta_{-1}(\boldsymbol{r}) e^{i \omega_{\mathrm{L}} t}
\end{array}\right)
$$

where $\omega_{\mathrm{L}}$ is the Larmor frequency and $\zeta_{ \pm 1}$ depends only on $\boldsymbol{r}$ in the time scale of $\omega_{\mathrm{L}}^{-1}$. For this state, $F_{y} \simeq 0$, and the phase-contrast signal is proportional to

$$
A_{0} n+\frac{1}{2} A_{2}\left[\left|\zeta_{1}\right|^{2}+\left|\zeta_{-1}\right|^{2}+2 \operatorname{Re}\left(\zeta_{1}^{*} \zeta_{-1} e^{2 i \omega_{L} t}\right)\right]
$$

From this signal oscillating at the frequency $2 \omega_{\mathrm{L}}$, we can determine the spatial distribution of the relative phase between the $m= \pm 1$ components. Around a half-quantum vortex, the phase of the oscillating signal changes by $2 \pi$. The ratio of the oscillating signal to the bias is proportional to

$$
R=\frac{A_{2}}{A_{0}+A_{2} / 2} .
$$

For the present purpose, therefore, the $F=1 \rightarrow F^{\prime}=0$
$\mathrm{D}_{2}$ transition may be most suitable, since $R$ is largest (Tab. I).

\section{CONCLUSIONS}

We have proposed a method to create a spin-dependent optical potential using near-resonant circularly polarized laser beams. We have shown that spin vortices can be nucleated in a spinor BEC using the spin-dependent rotating potential.

We considered a situation in which only the $m=1$ component of the antiferromagnetic ground state of a spin- ${ }^{23} \mathrm{Na}$ BEC is stirred by the spin-dependent rotating potential, and found that half-quantum vortices enter the condensate. To our knowledge this is the first proposal for nucleating fractional vortices by a rotating stirrer. The spin-vortex nucleation occurs at a low rotation frequency $\left(\simeq 0.1 \omega_{\perp}\right)$, compared with vortex nucleation in a scalar BEC $\left(\simeq 0.7 \omega_{\perp}\right)$. Moreover, the spin vortices easily enter the condensate: the nucleation time is $t \simeq 50$ $\mathrm{ms}$ without dissipation. The spin vortices exit from the condensate, and the entry-exit cycles are repeated. We have also shown that the half-quantum vortices can be observed in a nondestructive manner using the method of the Berkeley group.

The spin-dependent optical potential is a powerful tool for manipulating a spinor BEC, and may be applied to the generation of various spin textures.

\section{Acknowledgments}

We thank S. Tojo for valuable comments from the experimental point of view. This work was supported by the Ministry of Education, Culture, Sports, Science and Technology of Japan (Grants-in-Aid for Scientific Research, No. 17071005 and No. 20540388) and by the Matsuo Foundation.
[1] M. R. Matthews, B. P. Anderson, P. C. Haljan, D. S. Hall, C. E. Wieman, and E. A. Cornell, Phys. Rev. Lett. 83, 2498 (1999).

[2] A. E. Leanhardt, A. Görlitz, A. P. Chikkatur, D. Kielpinski, Y. Shin, D. E. Pritchard, and W. Ketterle, Phys. Rev. Lett. 89, 190403 (2002).

[3] M. F. Andersen, C. Ryu, P. Cladé, V. Natarajan, A. Vaziri, K. Helmerson, and W. D. Phillips, Phys. Rev. Lett. 97, 170406 (2006).

[4] K. W. Madison, F. Chevy, W. Wohlleben, and J. Dalibard, Phys. Rev. Lett. 84, 806 (2000).

[5] J. R. Abo-Shaeer, C. Raman, J. M. Vogels, and W. Ketterle, Science 292, 476 (2001).

[6] L. E. Sadler, J. M. Higbie, S. R. Leslie, M. Vengalattore, and D. M. Stamper-Kurn, Nature (London) 443, 312
(2006).

[7] J. M. Higbie, L. E. Sadler, S. Inouye, A. P. Chikkatur, S. R. Leslie, K. L. Moore, V. Savalli, and D. M. StamperKurn, Phys. Rev. Lett. 95, 050401 (2005).

[8] H. Saito, Y. Kawaguchi, and M. Ueda, Phys. Rev. Lett. 96, 065302 (2006); Phys. Rev. A 75, 013621 (2007).

[9] A. E. Leanhardt, Y. Shin, D. Kielpinski, D. E. Pritchard, and W. Ketterle, Phys. Rev. Lett. 90, 140403 (2003).

[10] T. Mizushima, K. Machida, and T. Kita, Phys. Rev. Lett. 89, 030401 (2002); T. Mizushima, N. Kobayashi, and K. Machida, Phys. Rev. A 70, 043613 (2004).

[11] K. W. Madison, F. Chevy, V. Bretin, and J. Dalibard, Phys. Rev. Lett. 86, 4443 (2001).

[12] A. Recati, F. Zambelli, and S. Stringari, Phys. Rev. Lett. 86, 377 (2001). 
[13] S. Sinha and Y. Castin, Phys. Rev. Lett. 87, 190402 (2001).

[14] M. Tsubota, K. Kasamatsu, and M. Ueda, Phys. Rev. A 65, 023603 (2002).

[15] G. E. Volovik and V. P. Mineev, JETP Lett. 24, 561 (1976).

[16] U. Leonhardt and G. E. Volovik, JETP Lett. 72, 46 (2000).

[17] J. Ruostekoski and J. R. Anglin, Phys. Rev. Lett. 91, 190402 (2003).

[18] T.-L. Ho, Phys. Rev. Lett. 81, 742 (1998).
[19] D. M. Stamper-Kurn, M. R. Andrews, A. P. Chikkatur, S. Inouye, H.-J. Miesner, J. Stenger, and W. Ketterle, Phys. Rev. Lett. 80, 2027 (1998).

[20] J. Stenger, S. Inouye, D. M. Stamper-Kurn, H.-J. Miesner, A. P. Chikkatur, and W. Ketterle, Nature (London) 396, 345 (1998).

[21] A. Crubellier, O. Dulieu, F. Masnou-Seeuws, M. Elbs, H. Knöckel, and E. Tiemann, Eur. Phys. J. D 6, 211 (1999).

[22] A. T. Black, E. Gomez, L. D. Turner, S. Jung, and P. D. Lett, Phys. Rev. Lett. 99, 070403 (2007). 